\title{
Letters
}

\section{Troglitazone decreases serum uric acid concentrations in Type II diabetic patients and non-diabetics}

\section{Dear Sir,}

Hyperuricaemia is commonly seen in association with obesity, impaired glucose tolerance, hypertension and dyslipidaemia $[1,2]$, which are the central characteristics of the insulin resistance syndrome. The association between isolated hyperuricaemia and insulin resistance is, however, still not defined [3]. To assess whether or not hyperuricaemia is an expression of insulin resistance, it seems reasonable to measure alterations in serum uric acid concentrations during treatment with an insulin sensitising agent in patients with Type II (non-insulin-dependent) diabetes mellitus. With this aim, we recruited 95 (61 men) Type II diabetic patients who were given $200 \mathrm{mg}$ of troglitazone twice a day and met the following criteria: no use of drugs that affect uric acid metabolism, no increase in serum creatinine concentration $(<115 \mu \mathrm{mol} / \mathrm{l})$ and availability of paired determinations of serum uric acid concentrations before and after troglitazone treatment. Their age (mean \pm SD) was $61.1 \pm 10.3$ years and BMI was $25.4 \pm 3.0$. All medications other than troglitazone (if any) were unchanged throughout the study period of $9.7 \pm 7.6$ months. All available data from 6 months before troglitazone and all data after it were combined respectively and used for statistical analysis by Student's paired $t$-test. Serum uric acid concentrations decreased significantly after treatment with troglitazone from a mean baseline value of $327 \pm 71 \mu \mathrm{mol} / 1$ to $298 \pm 71 \mu \mathrm{mol} / 1(p<0.0001): 351 \pm$ 71 to $321 \pm 65$ in men, $286 \pm 54$ to $244 \pm 48$ in women (both $p<0.0001$ ) (Fig. 1). This decrease occurred concomitantly with those in $\mathrm{HbA}_{1 \mathrm{c}}$ values $(8.4 \pm 1.3$ to $7.8 \pm 1.3 \%, p<$ $0.0001)$ and triglyceride concentrations $(2.2 \pm 1.3$ to $1.9 \pm$ $1.2 \mathrm{mmol} / 1, p<0.01)$ and in a subset of patients $(n=34)$ in parallel with fasting plasma glucose $(10.5 \pm 2.1$ to $8.3 \pm 2.4 \mathrm{mmol} / \mathrm{l}$, $p<0.0001)$ and insulin concentrations $(52.2 \pm 22.2$ to $34.8 \pm$ $15.0 \mathrm{pmol} / \mathrm{l}, p<0.0001)$ and therefore also HOMA-IR, a simple measure of insulin resistance obtained by fasting plasma

Corresponding author: Dr T. Wasada, Diabetes Center, Tokyo Women's Medical University, School of Medicine, 8-1 Kawada-cho, Shinjuku-ku, Tokyo 166-8666, Japan concentration of glucose $(\mathrm{mmol} / \mathrm{l}) \times$ insulin $(\mu \mathrm{U} / \mathrm{ml}) / 22.5$ $(4.3 \pm 2.4$ to $2.2 \pm 1.2, p<0.0001)$. Furthermore, in six non-diabetic subjects (two healthy volunteers and four hyperuricaemic patients) who consented to participate in a troglitazone trial (200 mg/day for 4 weeks), serum uric acid decreased similarly $(375 \pm 60$ to $298 \pm 30 \mu \mathrm{mol} / 1, p<0.02)$. A 24 -h urinary excretion of uric acid $(3.8 \pm 1.1$ to $3.3 \pm 0.8 \mathrm{mmol} /$ day $)$, uric acid clearance $(9.0 \pm 4.0$ to $8.3 \pm 3.3 \mathrm{ml} / \mathrm{min})$, and the ratio of uric acid clearance to that of creatinine $(10.0 \pm 4.3$ to $11.0 \pm 5.0 \%)$ determined in 12 Type II diabetic patients did not differ significantly between pre-treatment and post-treatment. It is therefore difficult to explain the decrease in serum uric acid concentrations given previous reports that hyperinsulinaemia reduces renal clearance of uric acid through coupling with increased sodium reabsorption at proximal tubules [4-7]. Haemodilution seems unlikely, because haematocrit values did not alter greatly with the treatment (data not shown). In addition, improvement of glycaemic control is not the cause because urinary excretion of uric acid is facilitated by hyperglycaemia itself and vice versa. Although the mechanism is not clear, we speculate that troglitazone could have some effect on uric acid production because increased purine biosynthesis and turnover with its attendant increase in serum uric acid concentrations due to increased activity of the hexose monophosphate shunt can be conceptually linked to insulin resistance or hyperinsulinaemia or both. Our results vary from another study [8] which showed no statistically significant alteration in serum uric acid concentrations or serum triglyceride, total-cholesterol and HDL-cholesterol, plasminogen activator inhibitor-1, conventional variables of insulin resistance, after troglitazone treatment (200 mg/day) in 25 Type II diabetic patients for 8 weeks using a randomised double-blind procedure. The reason for this disparity is not clear but is presumably due to differences in the dosage used in both studies: theirs was half of ours. It is not clear from this in vivo study whether the hypouricaemic effect of troglitazone is a direct pharmacological action or an indirect one secondary to reduction of insulin resistance. We favour the latter, however, because biochemical variables for insulin resistance changed concomitantly with serum uric acid concentrations in our study but the lack of changes in these variables was not accompanied by alterations in serum uric acid concentrations in the other study [8]. Such an effect has not been reported in the other thiazolidinediones, pioglitazone and rosiglitazone. We showed an antihyperuricaemic effect of troglitazone through a mechanism distinct from increased renal clear- 


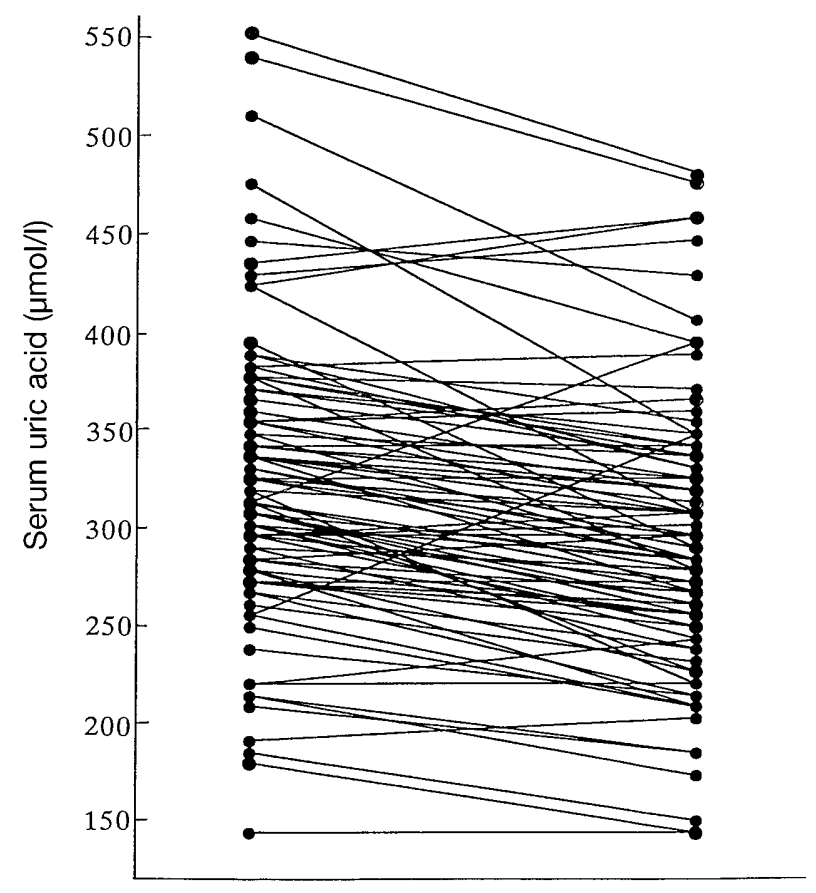

Pre-treatment

Post-treatment

Fig.1. Change in serum uric acid concentrations after treatment with troglitazone in 95 Type II diabetic patients

ance in Type II diabetic and in non-diabetic subjects. These findings again support the close association between hyperuricaemia and insulin resistance or hyperinsulinaemia or both.

Yours faithfully,

M. Iwatani, T. Wasada, K. Katsumori, C. Watanabe-Takahashi, N. Kamatani, Y. Iwamoto

\section{References}

1. Modan M, Halkin H, Karasik A, Lusky A (1987) Elevated serum uric acid - a facet of hyperinsulinaemia. Diabetologia 30: 713-718

2. Facchini F (1991) Relationship between resistance to insulin-mediated glucose uptake, urinary uric acid clearance, and plasma uric acid concentration. JAMA 266: 3008-3011

3. Bonora E, Kiechl S, Willeit J et al. (1998) Prevalence of insulin resistance in metabolic disorders, the Bruneck Study. Diabetes 47: 1643-1649

4. Quinones GA, Natali A, Baldi S et al. (1995) Effect of insulin on uric acid excretion in humans. Am $\mathrm{J}$ Physiol 268:E1-E5

5. Muscelli E, Natali A, Bianchi S et al. (1996) Effect of insulin on renal sodium and uric acid handling in essential hypertension. Am J Hypertens 9: 746-752

6. Vuorinen-Markkola H, Yki-Jarvinen H (1994) Hyperuricemia and insulin resistance. J Clin Endocrinol Metab 78: 25-29

7. Galvan AQ (1995) Effect of insulin on uric acid excretion in humans. Am J Physiol 268:E1-E5

8. Sironi AM, Vichi S, Gastaldelli A et al. (1997) Effects of troglitazone on insulin action and cardiovascular risk factors in patients with non-insulin-dependent diabetes. Clin Pharmacol Ther 62: 194-202

\section{Antibodies to tissue transglutaminase $\mathbf{C}$ in newly diagnosed and long-standing Type I diabetes mellitus}

\begin{abstract}
Dear Sir,
We read with interest the recent paper by Lampasona et al. [1] about the detection of autoantibodies to tissue transglutaminase $\mathrm{C}$ (tTGA) in patients with newly diagnosed Type I (insulin-dependent) diabetes mellitus to determine the extent of gluten-associated autoimmunity. Tissue transglutaminase $\mathrm{C}$ has been recently identified as an autoantigen target of antiendomysium antibodies (EMA), known as the serological marker of coeliac disease (CD) [2]. The authors found a prevalence of $\mathrm{IgA}$ antibodies to transglutaminase $\mathrm{C}$ [1] in about $8 \%$ and a low level of IgG autoantibodies to transglutaminase $\mathrm{C}$ in a further $32 \%$ of newly diagnosed Type I diabetic patients. This suggests that the high prevalence of autoimmunity to transglutaminase $\mathrm{C}$ could be due to an involvement of the gut in the pathogenesis of Type I diabetes or to a release of trans-
\end{abstract} glutaminase $\mathrm{C}$ from destroyed pancreatic beta cells.

We report our results about the detection of specific IgA to tTG and IgA antiendomysium (EMA) in 68 patients with Type I diabetes ( 36 males and 32 females, aged between 1 and 25.7 years). Amongst patients, 31 were newly diagnosed and 37 had long-standing diabetes, with a disease duration ranging between 1.1 and 16 years. Antibodies to protein tyrosine phosphatase (IA-2A) and to glutamic acid decarboxylase (GADA) were also detected in all patients. We measured tTG-IgA by an immunoenzymatic method (Genesis Diagnostics, Cambridgeshire, UK); both intra-assay and inter-assay coefficients of variation of this test were less than $12 \%$. We detected EMA by immunofluorescence (Bios Labordiagnostik, Grafelfing, Germany), IA-2A and GADA by radioimmunoassay (CIS Bio International - ORIS Group - Cedex, F and Medipan Diagnostica, Solchow, Germany, respectively). We defined tTGIgA values higher than $14 \mathrm{U} / \mathrm{ml}$ (mean $+3 \mathrm{SD}$ of 56 age and sex-matched healthy control subjects) as positive. Out of 68 patients $18(26.4 \%)$, in particular 6 newly diagnosed $(19.3 \%)$ and 12 with long-standing diabetes $(32.4 \%)$, were positive for tTG$\operatorname{IgA}$, with a higher frequency than control subjects $(0 / 56)$ (Yates' corrected $\chi^{2}=15.27 ; p=0.000093$ ). The high frequency of tTG-IgA found in our newly diagnosed patients is different from the results in other reports $[1,4]$. This could be due to the different method used for tTG-IgA detection (radioimmunoassay instead of enzyme-linked immunoassay) [1, 4]. Amongst 31 newly diagnosed patients, $6(19.3 \%)$ were positive for tTGA, and 2 of them for both tTGA and EMA. Jejunal biopsy in these 2 patients with both tTGA and EMA showed coeliac disease. Amongst 37 patients with long-standing diabetes, $12(32.4 \%)$ were positive for tTG-IgA and 10 of them for both tTG-IgA and EMA. Jejunal biopsy in these 10 patients showed total villous atrophy compatible with coeliac disease in 9 and partial villous atrophy compatible with "latent" coeliac disease in 1.

Immunological markers of Type I diabetes showed a significant association between GADA positivity and antibodies associated with coeliac disease in long-standing patients $\left(p=0.0006, \chi^{2}\right.$ test $)$, not found in newly diagnosed patients (NS, Fisher exact test) (Table 1). It has been reported [5] that GADA detection in patients with long-standing diabetes could be due to a persistence of some residual beta cells or specific environmental factors or both, capable of sustaining the au-

Corresponding author: R. Lorini, MD, Department of Paediatrics, G. Gaslini Institute, Largo Gaslini 5, 16147 Genova Quarto, Italy 